\title{
Dynamic signatures of electronically nonadiabatic coupling in sodium hydride: a rigorous test for the symmetric quasi-classical model applied to realistic, ab initio electronic states
}

\author{
Justin J. Talbot ${ }^{1 *}$, Martin Head-Gordon ${ }^{1,2}$, William H. Miller ${ }^{1}$, and \\ Stephen J. Cotton ${ }^{1}$ \\ ${ }^{1}$ Department of Chemistry, University of California, Berkeley, California 94720, \\ United States \\ ${ }^{2}$ Chemical Sciences Division, Lawrence Berkeley National Laboratory, Berkeley, \\ California, 94720, United States \\ *Email: justin.talbot@berkeley.edu
}

\begin{abstract}
Sodium hydride $(\mathrm{NaH})$ in the gas phase presents a seemingly simple electronic structure making it a potentially tractable system for the detailed investigation of nonadiabatic molecular dynamics from both computational and experimental standpoints. The single vibrational degree of freedom, as well as the strong nonadiabatic coupling that arises from the excited electronic states taking on considerable ionic character, provides a realistic chemical system to test the accuracy of quasi-classical methods to model population dynamics where the results are directly comparable against quantum mechanical benchmarks. Using a simulated pump-probe experiment, this work presents computational predictions of population transfer through the avoided crossings of $\mathrm{NaH}$ via symmetric quasi-classical Meyer-Miller (SQC/MM), Ehrenfest, and exact quantum
\end{abstract}


dynamics on realistic, ab initio potential energy surfaces. The main driving force for population transfer arises from a sharply localized avoided crossing between the $\mathbf{C}^{1} \Sigma^{+}$ and $\mathbf{D}^{1} \Sigma^{+}$potential energy surfaces which causes most of the population to transfer between $t=15$ and $t=30$ fs depending on the initially excited vibronic wavepacket. While quantum mechanical effects are expected due to the reduced mass of $\mathrm{NaH}$, predictions of the population dynamics from both the SQC/MM and Ehrenfest models perform remarkably well against the quantum dynamics benchmark. Additionally, an analysis of the vibronic structure in the nonadiabatically coupled regime and predicted transient absorption signatures are presented using a variational eigensolver methodology. The prospects for complementary experimental measurements are also assessed.

\section{Introduction}

Electronically nonadiabatic processes are ubiquitous throughout many important areas of chemistry. ${ }^{1-6}$ In general, dynamic predictions of these processes requires the calculation of a set of potential energy surfaces, nuclear gradients, and nonadiabatic coupling vectors, as well as a time propagation model for the electronic and nuclear degrees-of-freedom (DOF). In the dynamics context, one typically requires analytic gradients for the nuclear forces and nonadiabatic couplings which, collectively, can amount to by far the dominant computational expense. However, over the last decade, ab initio electronic structure theory has seen substantial progress in the efficient and accurate calculation of nonadiabatic couplings either through diabatization models ${ }^{7-11}$ or directly as a first-order derivative coupling vector in

the adiabatic representation. ${ }^{12-16}$ Providing meaningful estimates of nonadiabatic coupling, however often results in a trade-off between accurate wavefunction-based electronic structure approaches, which can include electron correlation but create a substantial computational cost, or more approximate electronic structure theories which allow for the study of larger systems with higher complexity.

Many dynamics methods have been developed already which can, in principle, utilize an $a b$ initio treatment of the electronic structure when propagating the electronic and nuclear 

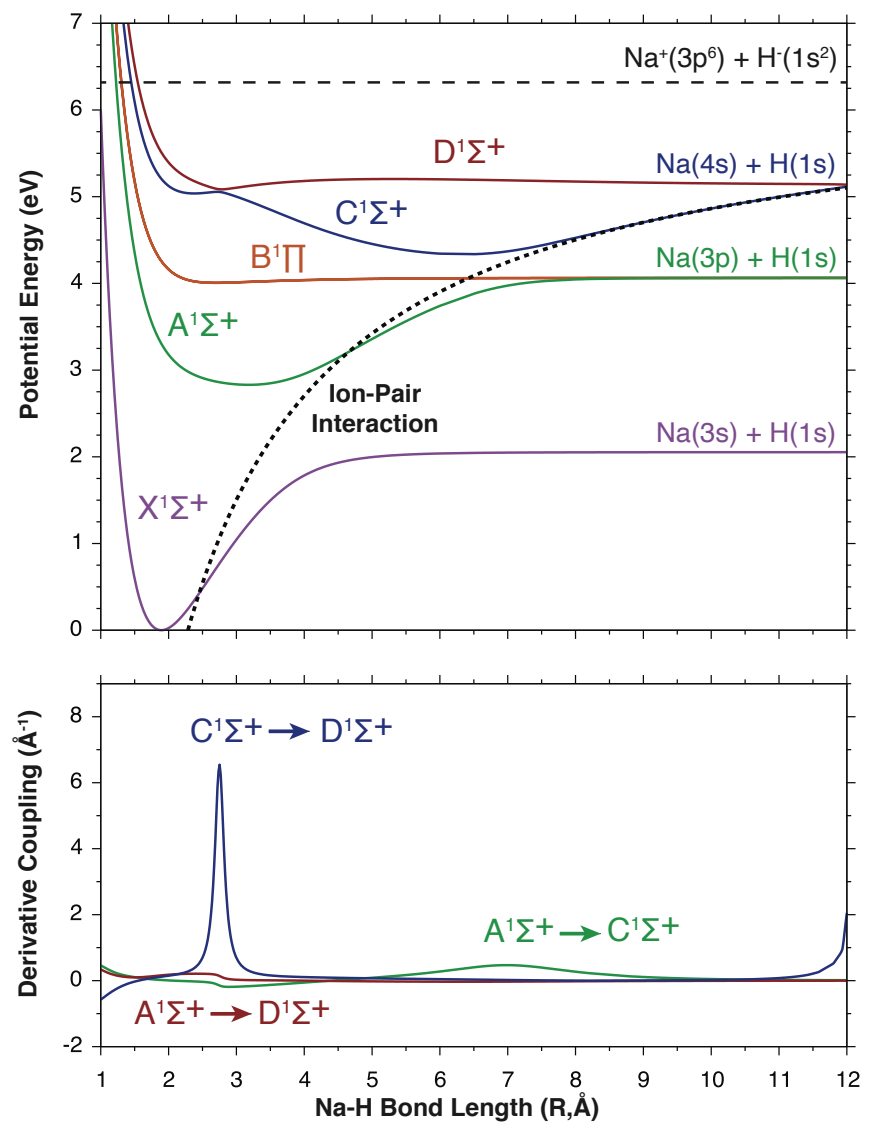

Fig. 1: A comparison between the potential energy surfaces (this work) and the ion-pair interaction energy (dotted line) of the lowest-energy singlet electronic states of sodium hydride (top). The dashed line at $6.3 \mathrm{eV}$ represents the ion-pair dissociation limit. The calculated bond length dependence of the first-order derivative couplings between the excited singlet states (bottom). The strongest but most localized first-order derivative coupling occurs between the C-D potential energy surfaces (blue) while a much weaker first-order derivative coupling exists between the A-C (green) and the A-D potential energy surfaces (brown).

DOF (FSSH, Ehrenfest, AIMS, SQC/MM, etc.) which all involve different approximations and cost trade-offs. ${ }^{17,18}$ Ideally, one would choose a quantum mechanical-based method for both the electronic and nuclear dynamics, which generally offers the greatest accuracy, but as these methods scale exponentially with system size, they are typically prohibitively expensive for all but the lowest-dimensional chemical systems. ${ }^{19-21}$ When true quantum mechanical effects, i.e., tunneling, are not too important treating the electronic and/or nuclear DOF with classical mechanics offers an appealing low cost alternative with easily parallelizable trajectories that are directly amenable to electronic structure calculations.

The symmetric quasi-classical Meyer-Miller (SQC/MM) approach is in essence a simple 
quantization model used in conjunction with the classical electronic+nuclear dynamics arising from the Meyer-Miller (MM) Hamiltonian. ${ }^{22-29}$ As such, SQC/MM has predicted with reasonable accuracy many different electronically nonadiabatic processes for a wide range of model systems, from the small coupling to the large coupling regime, while maintaining a foundation that is based entirely in classical mechanics. ${ }^{30,31}$ While the treatment of model chemical systems with SQC/MM have oftentimes been demonstrated in the diabatic representation, for $a b$ initio simulations, the natural representation is in terms of the adiabatic electronic states arising from the Born-Oppenheimer approximation, and recent years have seen significant progress in the development and application of SQC/MM to study general molecular systems in the adiabatic basis where potential energy surfaces, nuclear gradients, and nonadiabatic coupling vectors are calculated "on-the-fly" using available electronic structure theories. ${ }^{24,32-35}$ Pushing these ideas further, demonstrative calculations of the SQC/MM model where the results are readily comparable against experimental measurements and/or quantum mechanical benchmarks, represents another step towards the development of a sufficiently accurate "black box" approach for predicting the nonadiabatic dynamics of general molecular systems.

Alkali hydrides are some of the simplest diatomic molecules which may serve as realistic $a b$ initio test systems for studying the accuracy of the SQC/MM model. Their ground electronic states $\left(\mathbf{X}^{1} \Sigma^{+}\right)$are primarily single configurational, with energies well-separated from their excited electronic states. Likewise, their deep potential wells lend themselves to small anharmonicity constants, where calculations of ground state properties qualitatively reproduce experimental observations, even with crude theoretical models. ${ }^{36-40}$ In their excited electronic states, however, these seemingly simple systems gain substantial complexity as their electronic structure at longer bond lengths takes on a considerable amount of ionic character. ${ }^{41,42}$ This, in turn, introduces a cascade of avoided crossings between the excited Born-Oppenheimer potential energy surfaces. Since the low-lying electronic states of these systems are generally well-described with only single and double excitations, in a manageable active space, they are also prime candidates for the use of accurate wavefunction 
theories like equation-of-motion coupled cluster theory with single and double excitations (EOM-EE-CCSD). The benefit of using such a high level of electronic structure theory for a such simple systems is that a quantitatively accurate description of the underlying potential energy surfaces may be achieved, along with accurate analytic gradients (for the calculation of nuclear forces) and nonadiabatic derivative-coupling vectors. ${ }^{43-45}$

While to some degree all of the alkali hydrides exhibit this series of avoided crossings, a prime example is seen in the excited potential energy surfaces of sodium hydride (see Fig. 1). ${ }^{46}$ The relatively low-energy, ion-pair dissociation limit, $\mathrm{Na}^{+}\left(3 \mathrm{p}^{6}\right)+\mathrm{H}^{-}\left(1 \mathrm{~s}^{2}\right)$, introduces significant anharmonicity along the $\mathbf{A}, \mathbf{C}$, and $\mathbf{D}^{1} \Sigma^{+}$potential energy surfaces as their outer wells take on a substantial amount of ionic character. For the $\mathbf{A}$ electronic state, the ionic contribution extends the outer well to long bond lengths, in effect, forming a shallow potential well that dissociates to the $\mathrm{Na}(3 \mathrm{p})+\mathrm{H}(1 \mathrm{~s})$ limit. As the ion-pair dissociation limit is approached by the $\mathbf{C}$ and $\mathbf{D}$ potential energy surfaces however, the ionic character becomes more pronounced creating an outer well of the $\mathbf{C}$ electronic state that is purely ionic. This, in effect, forms a delocalized double-well potential in the $\mathbf{C}$ electronic state with a sharply localized avoided crossing between the $\mathbf{C}$ and $\mathbf{D}$ potential energy surfaces (see the bottom panel of Fig. 1). When this double-well potential is formed, the displacement of the $\mathbf{C}$ surface's outer well introduces an additional, although far weaker, avoided crossing with the $\mathbf{A}$ surface. Finally, while the $\mathbf{C}$ state dissociates to the $\mathrm{Na}(4 \mathrm{~s})+\mathrm{H}(1 \mathrm{~s})$ asymptote, the $\mathbf{D}$ state is known to dissociate to the ion-pair limit introducing yet another avoided crossing between the $\mathbf{C}$ and $\mathbf{D}$ states at a displaced $\mathbf{R}=12 \AA$ bond length.

Recent studies of sodium hydride have focused on utilizing available experimental and theoretical data to fit quantitatively accurate ground and excited state potential energy surfaces. On the theoretical front, a study by Aymar, Deiglmayr, and Deliu found that static polarizibilities, transition, and permanent dipole moments could be accurately predicted by modelling the electronic structure of sodium hydride as an effective two-electron system in the full configuration interaction limit - solidifying the use of double excitation methods for a quantitative description of electronic properties. ${ }^{46}$ Later, using these calculations as well 
as available experimental data, Walji, Sentjens, and Le Roy went on to fit highly accurate potential energy surfaces for the $\mathbf{X}$ and $\mathbf{A}$ states capable of producing vibrational lines that are directly comparable to experimental measurements. ${ }^{47,48}$ From experiments, a study from $\mathrm{Chu}$, et al. found the double-well potential of the $\mathbf{C}$ state could be accessed using opticaloptical double resonance fluorescence spectroscopy. ${ }^{49,50}$ After a potential fit analysis, the highest experimentally accessible vibrational level of the $\mathbf{C}$ state was determined which notably lies directly in the region of strong first-order derivative coupling between the $\mathbf{C}$ and $\mathbf{D}$ potential energy surfaces.

The aims of the present study are two-fold. First, electronic structure, vibronic energy levels, and predicted transient absorption signatures of the low-lying singlet electronic states of sodium hydride are presented with the hope that these benchmarks could be confirmed through a properly constructed experiment. These predictions were based from quantitatively accurate potential energy surfaces, analytic nuclear gradients, and first-order derivative couplings, as well as the vibronic eigenstates which were calculated using high-level electronic structure theory and a variational eigensolver. Second, using these calculations, the accuracy of SQC/MM to model the population dynamics through this series of avoided crossings is evaluated and compared against Ehrenfest predictions and quantum wavepacket benchmarks.

\section{Methods}

\subsection{Electronic Structure}

The potential energy surfaces, analytic nuclear gradients, and first-order derivative couplings were calculated using the EOM-EE-CCSD method with the core-valence polarized aug-ccpCVQZ basis set. The electronic basis set was chosen from the convergence behavior after increasing angular momentum in the basis set. The results of the convergence tests are

provided in the ESI. ${ }^{\dagger}$ Both the excitation energies, as well as four Rydberg states of the sodium atom were investigated. The number of occupied orbitals included in the correlated 
calculations, between frozen-core and core-valence polarized basis sets, was determined from convergence tests of the EOM-EE-CCSD excitation energies evaluated at the ground state equilibrium bond length. Correlation consistency was obtained after omitting the sodium atom's $1 s$ orbital from the correlated calculations. Likewise, core-valence polarized basis functions were required to obtain a reasonable agreement with available experimental data for the Rydberg states of the sodium atom. The coupled-cluster expansion was truncated at double excitations as it was found that including a perturbative triples correction to the energy introduced only a slight improvement to the correlation energy of the $\mathbf{X}$ electronic state at the equilibrium bond length. All potential energies, analytic nuclear gradients, and first-order derivative couplings were calculated using a release version of the Q-Chem 5.3 software package. ${ }^{51}$

All electronic properties (energies, gradients, derivative couplings, oscillator strengths) were calculated on an evenly spaced grid along sodium hydride's normal-mode vibrational coordinate $(\mathbf{Q})$. The grid spacing was chosen as $0.026 \AA$. All first-order derivative coupling vectors were deduced from analytic gradients using Szalay's approach which is the standard method for calculating EOM-EE-CCSD first-order derivative couplings in Q-Chem 5.3. ${ }^{43,44}$ For a few significantly displaced bond lengths the coupled-cluster equations were non-convergent, and these points were removed from the data set. While there is substantial first-order derivative coupling between the $\mathbf{C}$ and $\mathbf{D}$ potential energy surfaces near $\mathbf{R}=12 \AA$, the grid was truncated as convergence of the coupled-cluster T-amplitudes was particularly problematic in this region. With the converged scan, all interior points were evaluated by interpolation using a linear spline. The bond length and normal mode dependence of the potential energy surfaces, nuclear gradients, and first-order derivative couplings are provided for reference in the ESI. ${ }^{\dagger}$

\subsection{Time-Independent Eigensolver}

In order to include the effects of first-order derivative coupling on the vibrational energy levels, the vibronic Schrödinger equation ${ }^{4}$ was solved numerically using a modified Fourier- 
$\operatorname{grid}^{52}$ variational eigensolver and the Hamiltonian shown in Eq 1:

$$
\hat{\mathbf{H}}_{i j}=\frac{1}{2 \boldsymbol{\mu}}(\hat{\mathbf{P}}-i \hbar \mathbf{d}(\mathbf{Q}))_{i j}^{2}+E_{i}(\mathbf{Q}) \delta_{i j}
$$

where $\boldsymbol{\mu}$ is the normal-mode/reduced mass, $\hat{\mathbf{P}}_{i j}=-i \hbar \delta_{i j} \vec{\nabla}_{\mathbf{Q}, \mathbf{Q}}$, is the momentum operator expressed in the position representation, $\mathbf{d}(\mathbf{Q})$ is a skew-symmetric matrix of firstorder derivative coupling vectors, and $E_{i}(\mathbf{Q})$ is the potential energy surface corresponding to adiabatic state $i$. The vector-matrix $\mathbf{d}(\mathbf{Q})$ has elements $\mathbf{d}_{i j}(\mathbf{Q}) \equiv\left\langle\Phi_{i} \mid \nabla_{\mathbf{Q}} \Phi_{j}\right\rangle$ which are defined as the elements of the first-order derivative coupling vector between adiabatic Born-Oppenheimer electronic states $\Phi_{i}$ and $\Phi_{j}$ after projection onto the normal-mode co-

ordinate $\mathbf{Q}$. The momentum operator $\left(\hat{\mathbf{P}}_{i j}\right)$ was transformed from its diagonal momentum representation to the position representation using a forward and reverse Fourier transform accordingly. ${ }^{52}$ Solutions to the vibronic Schrödinger equation were computed variationally by expanding the eigenstates in the direct-product adiabatic basis shown in Eq. 2:

$$
\Psi(\mathbf{r}, \mathbf{Q})=\sum_{i} \Phi_{i}(\mathbf{r}, \mathbf{Q}) \chi_{i}(\mathbf{Q})
$$

where $\chi_{i}(\mathbf{Q})$ is the nuclear contribution to the adiabatic Born-Oppenheimer electronic state $\Phi_{i}(\mathbf{r}, \mathbf{Q})$. Only the $\mathbf{X}, \mathbf{A}, \mathbf{C}$, and $\mathbf{D}$ potential energy surfaces, and corresponding first-order derivative couplings were included in the calculation since coupling to the $\mathbf{B}^{1} \Pi$ state is symmetry forbidden. Due to the substantial grid size, integration of the potential energy surfaces and first-order derivative couplings required in total 2804 basis functions with 701 basis functions attributed directly to each adiabatic state. The Hamiltonian matrix was built and diagonalized using in-house code outside of Q-Chem. Adiabatic only calculations were performed using the same code by setting all matrix-vector elements $\mathbf{d}_{i j}(\mathbf{Q})=0$.

\subsection{SQC/MM Nonadiabatic Dynamics}

The SQC/MM approach to simulating nonadiabatic dynamics combines the classical Hamiltonian of Meyer and Miller (MM) with a simple symmetrical quasi-classical (SQC) quanti- 
zation procedure for defining the electronic state occupations and other observables.

In brief, the classical MM Hamiltonian ${ }^{29}$ maps the electronic DOF in a non-adiabatic process to a collection of classical harmonic oscillators, one for each of the electronic states. In a diabatic representation, it is given by

$$
H(\mathbf{x}, \mathbf{p}, \mathbf{Q}, \mathbf{P})=\frac{1}{2 \boldsymbol{\mu}} \mathbf{P}^{2}+\sum_{i}^{F}\left(\frac{1}{2} p_{i}^{2}+\frac{1}{2} x_{i}^{2}-\gamma_{i}\right) H_{i i}(\mathbf{Q})+\sum_{i<j}^{F}\left(p_{i} p_{j}+x_{i} x_{j}\right) H_{i j}(\mathbf{Q}),
$$

where $\left\{x_{i}, p_{i}\right\}$ are the coordinates and momenta of the "electronic oscillators" corresponding to a set of $F$ electronic states, $\mathbf{Q}, \mathbf{P}$ are the coordinates and momenta of the nuclear DOF having normal-mode/reduced masses $\boldsymbol{\mu},\left\{H_{i j}(\mathbf{Q})\right\}$ is an $F \times F$ nuclear coordinatedependent electronic matrix (diabatic in Eq. 3), and $\left\{\gamma_{i}\right\}$ are a set of zero point energy (ZPE) parameters which are initially adjusted per DOF as noted below. The evolution of the $F$ classical oscillators in Eq. 3 thus describes the electronic configuration in the MM model and, in particular, the classical actions associated with each oscillator

$$
n_{i} \equiv \frac{1}{2} p_{i}^{2}+\frac{1}{2} x_{i}^{2}-\gamma_{i}
$$

represent the electronic occupations. The actions $\left\{n_{i}\right\}$ are specifically what are quantized via the SQC windowing protocol ${ }^{22,23}$, by multiplicatively weighting the potential energy surfaces, $\left\{H_{i i}(\mathbf{Q})\right\}$ in Eq. 3, they determine the effective forces on the nuclei.

In realistic simulations employing rigorous ab initio electronic structure theory, the resulting electronic states are adiabatic and, in the adiabatic representation, the MM Hamiltonian is given by

$$
\mathbf{H}(\mathbf{x}, \mathbf{p}, \mathbf{Q}, \mathbf{P})=\frac{1}{2 \boldsymbol{\mu}}(\mathbf{P}+\Delta \mathbf{P}(\mathbf{x}, \mathbf{p}, \mathbf{Q}))^{2}+V_{\mathrm{eff}}(\mathbf{x}, \mathbf{p}, \mathbf{Q})
$$

where $\mathbf{P}$ is still a vector of canonical nuclear momenta but now arises in combination with 
a nonadiabatic coupling vector potential given by

$$
\Delta \mathbf{P}(\mathbf{x}, \mathbf{p}, \mathbf{Q})=\sum_{i<j}^{F}\left(x_{i} p_{j}-x_{j} p_{i}\right) \mathbf{d}_{i j}(\mathbf{Q})
$$

which depends explicitly on the standard first-derivative nonadiabatic coupling vector $\mathbf{d}_{i j}(\mathbf{Q}) \equiv$ $\left\langle\Phi_{i} \mid \nabla_{\mathbf{Q}} \Phi_{j}\right\rangle$ between adiabatic Born-Oppenheimer electronic states $\Phi_{i}$ and $\Phi_{j}$ (the electronic oscillator variables $\left\{x_{i}, p_{i}\right\}$ now corresponding to adiabatic electronic states). Eq. 5 also expresses the adiabatic MM Hamiltonian in terms of a symmetrized, occupation-weighted potential

$$
V_{\mathrm{eff}}(\mathbf{x}, \mathbf{p}, \mathbf{Q})=\frac{1}{F} \sum_{i}^{F} E_{i}(\mathbf{Q})+\frac{1}{F} \sum_{i<j}^{F}\left(n_{i}-n_{j}\right)\left(E_{i}(\mathbf{Q})-E_{j}(\mathbf{Q})\right)
$$

which is commonly employed in both adiabatic and diabatic calculations and guarantees the electronic dynamics are independent of energy scale. Of course, Eq. 7 only references the diagonal elements of the electronic matrix $\left\{E_{i}(\mathbf{Q}) \equiv H_{i i}(\mathbf{Q})\right\}$ because it is diagonal in the adiabatic representation.

Applying Hamilton's equations to Eqs. 5, 6, and 7 yields dynamically-consistent classical EOM for both nuclear and electronic DOF in terms of the canonical coordinates and momenta appearing in Eq. 5 but, unfortunately, will explicitly require the use of secondderivative non-adiabatic coupling matrices which are extremely impractical to calculate in a simulation employing realistic quantum chemistry for the electronic states (and not readily available in standard codes). The simple cure $^{24}$ is to re-write the canonical EOM in terms of a kinematic momentum, which results in kinematic EOM which contain only the first-derivative couplings $\mathbf{d}_{i j}$ but are nevertheless exactly equivalent; these kinematic EOM 
therefore represent the operational formulation employed exclusively in this work:

$$
\begin{aligned}
\dot{x}_{i} & =p_{i} \frac{1}{F} \sum_{j}^{F}\left(E_{i}(\mathbf{Q})-E_{j}(\mathbf{Q})\right)+\sum_{j}^{F} x_{j} \mathbf{d}_{j i}(\mathbf{Q}) \cdot \frac{\mathbf{P}_{\text {kin }}}{\mu}, \\
\dot{p}_{i} & =-x_{i} \frac{1}{F} \sum_{j}^{F}\left(E_{i}(\mathbf{Q})-E_{j}(\mathbf{Q})\right)+\sum_{j}^{F} p_{j} \mathbf{d}_{j i}(\mathbf{Q}) \cdot \frac{\mathbf{P}_{\text {kin }}}{\mu}, \\
\dot{\mathbf{Q}} & =\frac{\mathbf{P}_{\text {kin }}}{\mu} \\
\dot{\mathbf{P}}_{\text {kin }} & =-\frac{\partial V_{\text {eff }}}{\partial \mathbf{Q}}-\sum_{i j}\left(\frac{1}{2} p_{i} p_{j}+\frac{1}{2} x_{i} x_{j}\right)\left(E_{j}(\mathbf{Q})-E_{i}(\mathbf{Q})\right) \mathbf{d}_{i j}(\mathbf{Q}) .
\end{aligned}
$$

In the SQC/MM approach, quantization of the classical Hamiltonian dynamics produced by Eq. 8 is accomplished, initially and finally, by Monte Carlo sampling initial actions from a "windowing" function defined by the SQC model and, after running the dynamics for a prescribed time interval (via Eq. 8), "binning" the final actions by a symmetric windowing function. In this work, the triangle windowing model was chosen in combination with the $\gamma$-adjustment procedure, exactly as described in Ref. [25], except that here the adiabatic version of the MM Hamiltonian is employed through the EOM of Eq. 8. The key point of the $\gamma$-adjustment procedure is to set the $\left\{\gamma_{i}\right\}$ in Eq. 4 (and therefore in Eq. 7), per DOF (and per trajectory), so that, for each trajectory, the initial force on the nuclei is that of the initial pure quantum state -i.e., the single-surface force. With this prescription, the $\left\{\gamma_{i}\right\}$ still give the ensemble of trajectories an average ZPE of $\frac{1}{3}$ over the window function which, as described in the SQC/MM papers, is optimal in this model and somewhat less than the quantum value of $\frac{1}{2}$. Again, an important consequence of the SQC model in combination with the $\gamma$-adjustment protocol is that the nuclei are subject to ZPE fluctuations (in the forces from the electronic DOF) but that the dynamics is initiated with exactly singlesurface pure state forces corresponding to the initially excited adiabatic quantum state. 


\subsection{Transient Absorption and Population Dynamic Simulations}

The transient absorption spectrum and quantum/quasi-classical population dynamics were simulated assuming a pump-probe type experiment. The simulated experimental setup is as follows. At time $t=0$, a pump laser promotes the $\mathbf{X}$ ground vibronic state $(\mathbf{X}$, $\chi=0$ ), calculated either from the vibronic Schrödinger equation (quantum) or as the

harmonic oscillator ground vibrational state (quasi-classical) to the $\mathbf{A}$ potential energy surface which is then allowed to propagate for a delay time $\delta$. At select pump-probe time delays, between $0-250 \mathrm{fs}$, the pumped quantum wavepacket or classical particle moving on the A potential energy surface is promoted with a probe laser to either the $\mathbf{C}$ or $\mathbf{D}$ potential energy surfaces and allowed to further propagate. Finally, quantum and quasiclassical population dynamics, as well as the transient absorption spectrum of the quantum wavepacket, are recorded as the wavepacket or classical particle moves through the avoided crossing region between the $\mathbf{C}$ and $\mathbf{D}$ adiabatic states.

The transient absorption signatures were calculated quantum mechanically, up to $\delta=50$ fs in $\Delta \delta=5$ fs intervals, from the Franck-Condon overlap integrals between the quantum wavepacket, after promotion to the $\mathbf{C}$ or $\mathbf{D}$ potential energy surfaces, and the nonadiabatically coupled vibronic states of Eq. 2. The difference spectrum between the Franck-Condon factors after initial absorption $(\delta=0)$ and the Franck-Condon factors at time $\delta$ were broadened using a Lorentzian function with a linewidth of $100 \mathrm{meV}$ and the results were summed to give the separated component lineshapes. Then, using the computed vibronic energy levels, quantum wavepacket dynamics were performed by expanding the probed wavepacket as a linear combination of the nonadiabatically coupled eigenstates and this basis was propagated analytically by solving the time-dependent Schrödinger equation. Quantum dynamics were simulated, at each pump-probe delay time, for $t=60 \mathrm{fs}$ with a $0.1 \mathrm{fs}$ time step. Since the normalization of the wavepacket is a conserved quantity, the electronic state populations as a function of time were evaluated as the summed contribution to the total norm from each adiabatic basis function $\Phi_{i}$.

The Ehrenfest and SQC/MM dynamics were simulated by initially sampling 25, 000 
nuclear positions and momentum directly from a $0 K$ harmonic oscillator Wigner distribution. The reduced mass $(\mu=1.050 \mathrm{amu})$ and harmonic frequency $\left(\omega=1162.88 \mathrm{~cm}^{-1}\right)$ were calculated from the equilibrium bond length of the ground electronic state of $\mathrm{NaH}$. Coupled-cluster with single and double excitations and the aug-cc-pCVQZ basis set was used to calculate the harmonic frequency, reduced mass, and normal-vector $\mathbf{Q}$. In the dynamics, the Wigner sampled positions and momenta were allowed to propagate classically on the A potential energy surface, with a $0.1 \mathrm{fs}$ time step, for a time delay $\delta$, before being instantaneously promoted to the $\mathbf{C}$ or $\mathbf{D}$ potential energy surfaces where the particle was allowed to further propagate via Meyer-Miller multi-surface dynamics for $t=60 \mathrm{fs}$ with the same $0.1 \mathrm{fs}$ time step. The nuclear equations of motion were integrated numerically with a traditional velocity-Verlet integrator and a semi-analytic, direct diagonalization scheme was used to integrate the equations of motion for the electronic action variables. All dynamic simulations were performed using in-house code outside of Q-Chem. The Ehrenfest simulations were performed with the same code by setting $\gamma=0$.

\section{Results \& Discussion}

\subsection{Vibronic Level Structure}

The effect of first-order derivative coupling on the adiabatic vibrational energy levels is shown, for a few selected eigenvalues, in table 1 . The eigenstates were assigned to a single vibrational level $\chi_{i}$ on the $\mathbf{X}, \mathbf{A}, \mathbf{C}$, or $\mathbf{D}$ electronic states by taking the maximum squared projection onto the adiabatic basis functions $\chi_{i} \Phi_{i}$. The energy zero is set at the bottom of the well for the $\mathbf{X}$ potential energy surface which allows for direct comparison with Fig. 1.

As shown in the bottom panel of Fig. 1, the first-order derivative coupling between the $\mathbf{A}$ and $\mathbf{C}$ potential energy surfaces peaks at $\mathbf{R} \approx 7 \AA$ in bond length with a delocalized spread of nearly $\pm 2 \AA$. This weak coupling introduces minimal mixing with adiabatic states from the $\mathbf{C}$ manifold suggesting that the $\mathbf{A}$ vibrational levels maintain a near perfect adiabatic character - at least for low-lying vibrational levels. As the outer well of the A potential 

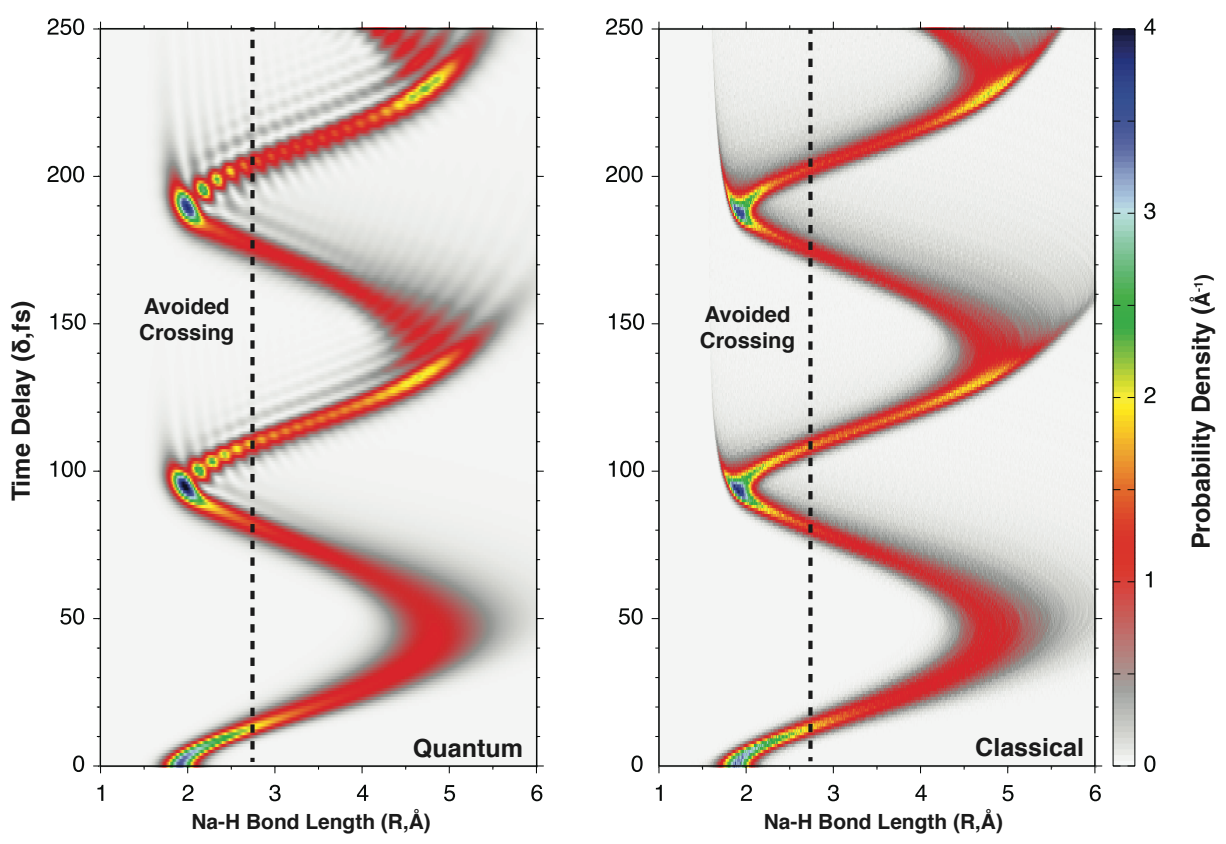

Fig. 2: The pump-probe delay time $\delta$ and bond length dependence of the quantum wavepacket (left) and the classical trajectories (right) propagating along the A potential energy surface. The dashed line in both plots is the position of maximum first-order derivative coupling between the $\mathbf{C}$ and $\mathbf{D}$ potential energy surfaces.

energy surfaces takes on substantial ionic character, the resulting anharmonicity creates a potential where the energetic splitting between vibrational levels slightly increases with vibrational excitation (see table 1). ${ }^{47}$ As a result, the low-lying vibrational eigenstates of the $\mathbf{A}$ state are delocalized over a wide range of bond lengths For example, the average bond length of the $\chi=8$ vibrational level is $\langle\mathbf{R}\rangle=3.41 \AA$ which is significantly longer than the $\mathbf{X}(\chi=0)$ ground state which only has an average bond length of $\langle\mathbf{R}\rangle=1.92 \AA$.

The prominent reduction in adiabatic character begins in the highly-excited vibrational levels of the $\mathbf{C}$ state and proceeds through the low-lying vibrational levels of the $\mathbf{D}$ state. In this regime, the vibrational states can no longer be considered adiabatic as there is a substantial amount of mixing that occurs between the adiabatic basis functions attributed to the $\mathbf{C}$ and $\mathbf{D}$ states. For example, the assignment of the ground vibrational level of the $\mathbf{D}$ adiabatic state, $(\mathbf{D}, \mathbf{0})$, has an adiabatic character of only 0.383 indicating that substantial mixing with nearby adiabatic vibronic states has occurred. While there is a sharp degradation in character for vibrational levels near the avoided crossing, the energetic 


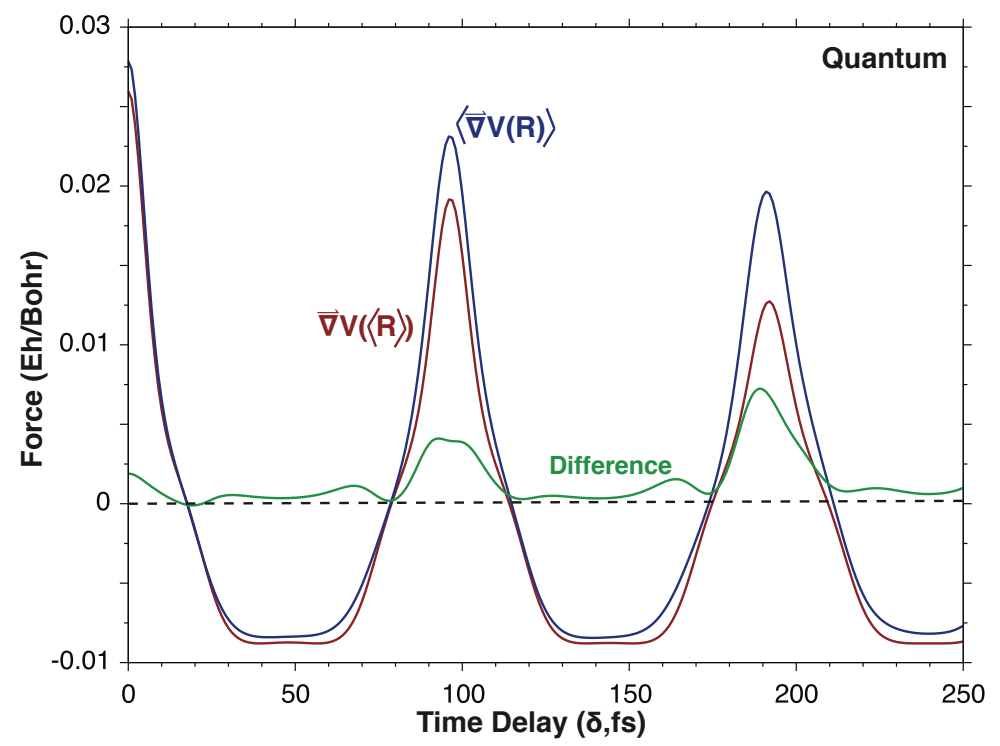

Fig. 3: A comparison between the average force and the force evaluated at the average position of the pumped quantum wavepacket propagating along the A potential energy surface as a function of pumpprobe delay time $\delta$.

shifts between the adiabatic states and their nonadiabatically coupled counterparts are surprisingly only on the order of $0.01 \mathrm{eV}$ which indicates that, while the states are still highly mixed, the effective first-order derivative coupling between the $\mathbf{C}$ and $\mathbf{D}$ potential energy surfaces remains localized to a small energetic region of the spectrum. A comparable degradation of adiabatic character also manifests in the low-lying vibrational levels of the D state with highly mixed states combined with a near negligible energy splittings between the adiabatic and nonadiabatically coupled states.

\section{2 $A^{1} \Sigma^{+}$Quantum/Classical Dynamics}

The time dependence of the initially excited quantum wavepacket $(\mathbf{X}, \chi=0)$, propagating along the A potential energy surface, is shown in the left panel of Fig. 2. The dashed line represents the bond length were the first-order derivative coupling between the $\mathbf{C}$ and $\mathbf{D}$ states is maximum-which occurs along the repulsive wall of the A potential energy surfaces. After initial promotion from the $\mathbf{X}$ state, there is a spreading of the quantum wavepacket that results from the anharmonicity of the $\mathbf{A}$ potential energy surface as the wavepacket 
Table 1: A comparison between selected adiabatic $\left(\mathbf{d}_{i j}(\mathbf{Q})=0\right)$ and nonadiabatically coupled vibronic energy levels and their assignments. Each nonadiabatically coupled eigenstate was assigned to a single vibrational level $\left(\chi_{i}\right)$ on a single adiabatic potential energy surface $\left(\Phi_{i}\right)$ by taking the maximum squared projection.

\begin{tabular}{|c|c|c|c|}
\hline State $\left(\Phi_{i}, \chi_{i}\right)$ & Adiabatic (eV) & Nonadiabatic (eV) & Character \\
\hline \multicolumn{4}{|c|}{$X^{1} \Sigma^{+}$} \\
\hline $\mathrm{X}, 0$ & 0.072 & 0.072 & 1.000 \\
\hline \multicolumn{4}{|c|}{$A^{1} \Sigma^{+}$} \\
\hline $\mathrm{A}, 0$ & 2.921 & 2.921 & 1.000 \\
\hline $\mathrm{A}, 1$ & 2.959 & 2.959 & 1.000 \\
\hline $\mathrm{A}, 2$ & 2.999 & 2.999 & 1.000 \\
\hline $\mathrm{A}, 3$ & 3.040 & 3.040 & 1.000 \\
\hline $\mathrm{A}, 4$ & 3.082 & 3.082 & 1.000 \\
\hline $\mathrm{A}, 5$ & 3.125 & 3.125 & 1.000 \\
\hline A, 6 & 3.168 & 3.168 & 1.000 \\
\hline $\mathrm{A}, 7$ & 3.212 & 3.212 & 1.000 \\
\hline $\mathrm{A}, 8$ & 3.255 & 3.256 & 1.000 \\
\hline \multicolumn{4}{|c|}{$C^{1} \Sigma^{+}$} \\
\hline $\mathrm{C}, 40$ & 5.183 & 5.181 & 0.842 \\
\hline $\mathrm{C}, 41$ & 5.197 & 5.199 & 0.653 \\
\hline $\mathrm{C}, 42$ & 5.211 & 5.214 & 0.742 \\
\hline $\mathrm{C}, 43$ & 5.226 & 5.232 & 0.363 \\
\hline $\mathrm{C}, 44$ & 5.241 & 5.247 & 0.414 \\
\hline $\mathrm{C}, 45$ & 5.258 & 5.264 & 0.476 \\
\hline $\mathrm{C}, 46$ & 5.275 & 5.282 & 0.382 \\
\hline $\mathrm{C}, 47$ & 5.292 & 5.300 & 0.399 \\
\hline $\mathrm{C}, 48$ & 5.309 & 5.315 & 0.182 \\
\hline $\mathrm{C}, 49$ & 5.327 & 5.318 & 0.236 \\
\hline \multicolumn{4}{|c|}{$D^{1} \Sigma^{+}$} \\
\hline $\mathrm{D}, 0$ & 5.187 & 5.187 & 0.383 \\
\hline $\mathrm{D}, 1$ & 5.223 & 5.223 & 0.803 \\
\hline $\mathrm{D}, 2$ & 5.230 & 5.230 & 0.844 \\
\hline $\mathrm{D}, 3$ & 5.231 & 5.222 & 0.253 \\
\hline $\mathrm{D}, 4$ & 5.238 & 5.238 & 0.992 \\
\hline $\mathrm{D}, 5$ & 5.246 & 5.246 & 0.705 \\
\hline $\mathrm{D}, 6$ & 5.254 & 5.254 & 0.973 \\
\hline $\mathrm{D}, 7$ & 5.259 & 5.251 & 0.436 \\
\hline $\mathrm{D}, 8$ & 5.261 & 5.261 & 0.979 \\
\hline
\end{tabular}

approaches the bottom of the $\mathbf{A}$ potential well. This spread maximizes between $\mathbf{R}=4 \AA$ and $\mathbf{R}=6 \AA$ in bond length which occurs as the wavepacket nears the classical turning point 
of the outer well of the A potential energy surface $(\delta \approx 50 \mathrm{fs})$. Then, as the inner well of the A potential energy surface is approached from longer bond lengths, a sharp contraction occurs as the quantum wavepacket moves up the repulsive wall. For pump-probe delay times longer that $90 \mathrm{fs}$, the spatial extent of the quantum wavepacket remains mostly intact although nodes are introduced due to further spreading. Throughout the 250 fs pump-probe delay range, the quantum wavepacket, moving on the A potential energy surface, passes through the avoided crossing region a total of five times - three from the left (shorter bond lengths) and two from the right (longer bond lengths).

Remarkably similar dynamics are seen when the motion on the A potential energy surface is purely classical and sampled from the $0 K$ harmonic oscillator Wigner distribution as shown in the right panel of Fig. 2. The classical motion on the A potential energy surface has a comparable spreading to the quantum wavepacket near the outer well, between $\mathbf{R}=4 \AA$ and $\mathbf{R}=6 \AA$, as well as a similar contraction as the particle approaches the repulsive wall near $\mathbf{R} \approx 2 \AA$. While both quantum and classical dynamics have a comparable period, wavelength, and general form across all pump-probe delay times, the primary distinction between the two approaches is the quantum wavepacket develops nodes at pump-probe delay times $\delta>100$ fs which aren't observed in the classical motion.

To investigate the near classical motion of the quantum wavepacket further, comparisons between the classical force averaged over the quantum wavepacket and the classical force evaluated at the quantum wavepacket's average position as a function of pump-probe time delay are provided in Fig. 3. The average force calculations suggest that since the initially excited quantum wavepacket is compact, due to the near-harmonic potential energy surface of the $\mathbf{X}$ electronic state, quantum motion on the $\mathbf{A}$ potential energy surface can be approximated, to a reasonable accuracy across all pump-probe delay times, with classical mechanics by Ehrenfest's theorem. 

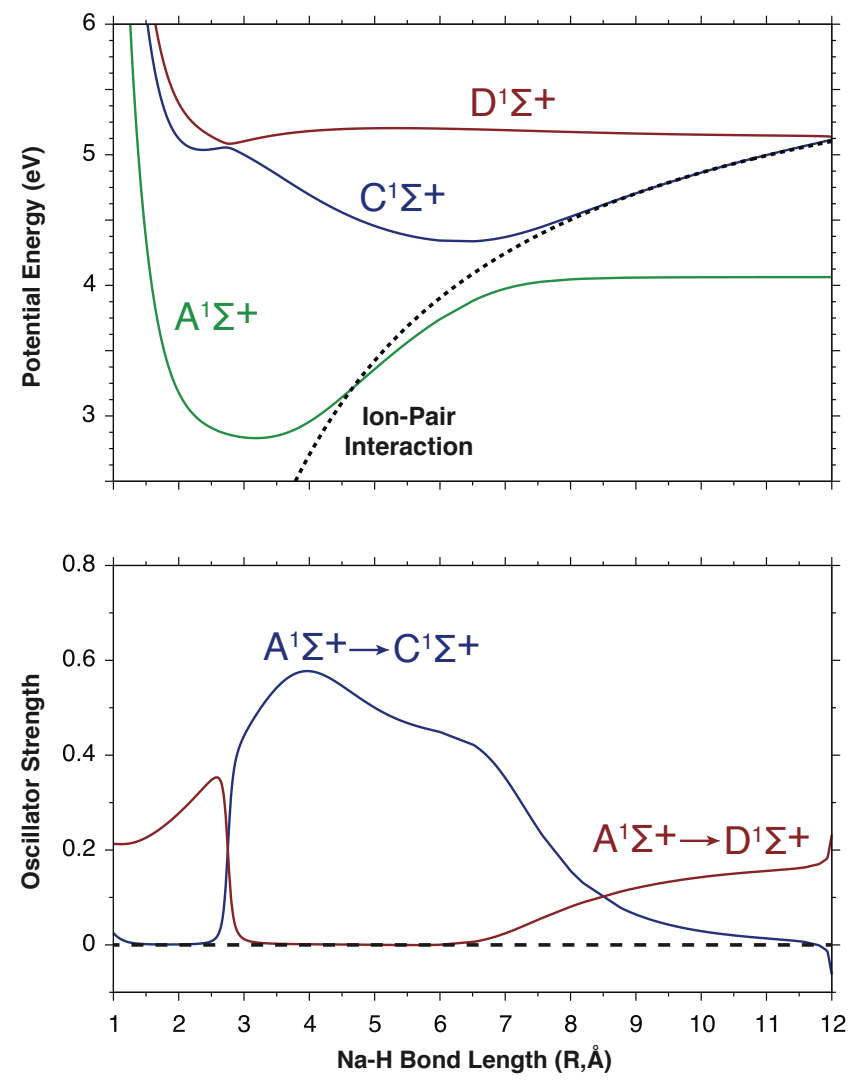

Fig. 4: The calculated A, C, and D potential energy surfaces (top). The bond length dependence of the oscillator strength between the $\mathbf{A} \rightarrow \mathbf{C}$ transition (blue) and $\mathbf{A} \rightarrow \mathbf{D}$ transition (brown) (bottom).

\subsection{Transient Absorption Spectrum}

As the pumped wavepacket is propagating along the A potential energy surface, the probability of absorption from the probe laser is determined by the oscillator strength of the $\mathbf{A} \rightarrow \mathbf{C}$ or $\mathbf{A} \rightarrow \mathbf{D}$ transitions. The bond length dependence on the oscillator strength is shown in the bottom panel of Fig. 4 with the $\mathbf{A}, \mathbf{C}$, and $\mathbf{D}$ potential energy surfaces shown for reference in the top panel. Noticeably, the full extent of the initial quantum wavepacket, at $t=0$, is entirely encompassed in the region where the oscillator strength is exclusively attributed to the $\mathbf{A} \rightarrow \mathbf{D}$ transition. However, as the wavepacket passes through the avoided crossing region for the first time, there is a dramatic change in character as the oscillator strength is now dominated by the $\mathbf{A} \rightarrow \mathbf{C}$ transition. The $\mathbf{A} \rightarrow \mathbf{C}$ oscillator strength peaks at $\mathbf{R} \approx 4 \AA$ before decaying at bond lengths $\mathbf{R}>7 \AA$. As even longer bond lengths are 

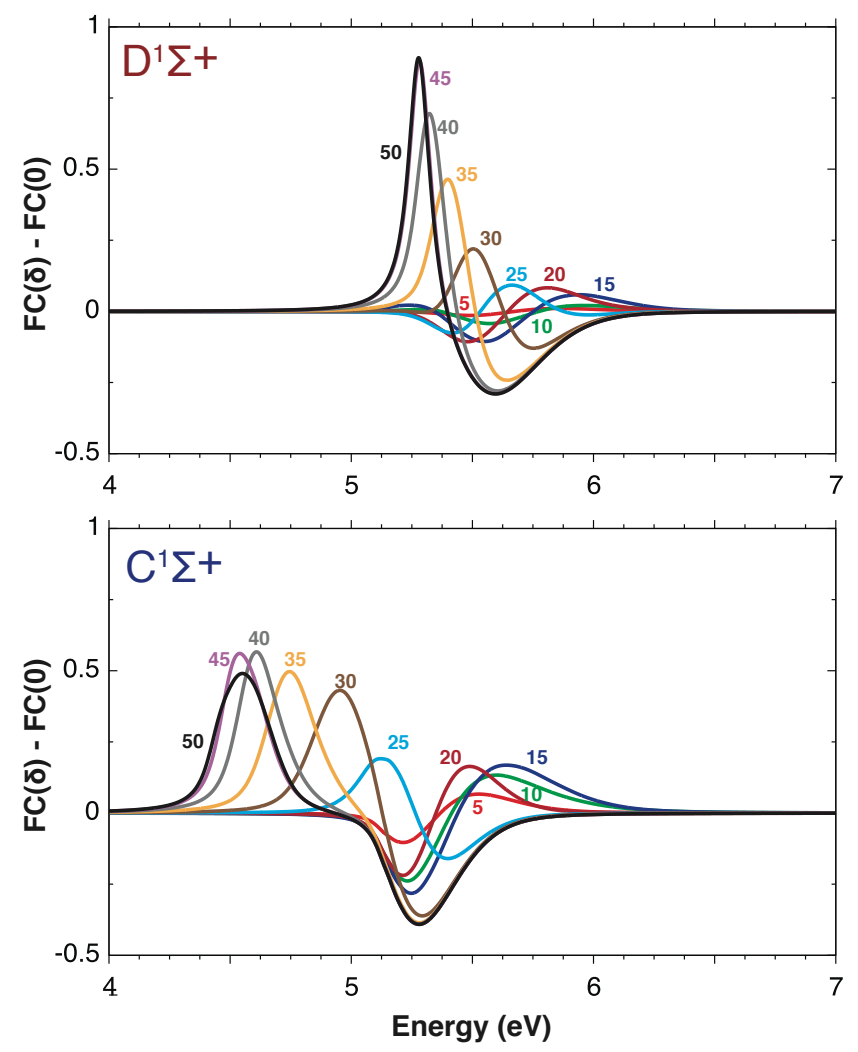

Fig. 5: The Franck-Condon simulated transient absorption spectrum. At delay times ranging from $5-50$ $\mathrm{fs}$, the initial quantum wavepacket $(\mathbf{X}, \chi=0)$, propagating on the $\mathbf{A}$ state, is promoted with a probe laser to the $\mathbf{C}$ (bottom) or $\mathbf{D}$ (top) potential energy surfaces. The Franck-Condon factors are broadened using a Lorentzian function with a linewidth of $100 \mathrm{meV}$ and summed.

approached, the overall oscillator strength is significantly reduced although there is an increase in $\mathbf{A} \rightarrow \mathbf{D}$ which results from approaching the $\mathbf{R} \approx 12 \AA$ avoided crossing between the $\mathbf{C}$ and $\mathbf{D}$ potential energy surfaces.

The Franck-Condon simulated transient absorption spectrum for the pumped quantum wavepacket, after promotion to the $\mathbf{C}$ state is shown in the bottom panel of Fig. 5. According to table 1, vibronic states in the avoided crossing region are between 5 and $6 \mathrm{eV}$. For pump-probe delay times between $\delta=5$ and $\delta=20 \mathrm{fs}$, both a weak excited state and ground state bleach signal is observed in this region indicating that initially the distribution is comprised primarily of vibronic states near the $\mathbf{C}-\mathbf{D}$ avoided crossing. For pump-probe delay times between $\delta=25$ and $\delta=50 \mathrm{fs}$, a ground state bleach signal greater than $5 \mathrm{eV}$, 
along with an excited state absorption signal for energies less than $5 \mathrm{eV}$, is observed which indicates that the distribution is now comprised of low-lying vibronic states attributed to the $\mathbf{C}$ state, far from the avoided crossing region which maintain most of their adiabatic character. Maximum intensity is reached at $\delta=40$ fs before the wavepacket turns around and begins approaching the avoided crossing region from the right.

The transient absorption spectrum after promotion to the $\mathbf{D}$ state presents a very different picture (see the top panel of Fig. 5). In this case, the entire transient absorption spectrum is above $5 \mathrm{eV}$. Consequentially, an intense excited state absorption signal isn't observed until $\delta=30$ fs which indicates that initially the distribution is comprised of vibronic energy levels that are near the avoided crossing and remain so for longer delay times when compared with promotion to the $\mathbf{C}$ state. The population transfer through the avoided crossing, after promotion to the $\mathbf{D}$ state will be, in general more efficient when compared to the quantum wavepacket promoted to the $\mathbf{C}$ state. Likewise, the ground state bleach signal is blue shifted compared to the $\mathbf{C}$ state indicating that the distribution is constructed primarily from low-lying vibronic states attributed to the $\mathbf{D}$ state. This suggests that population transfer through the avoided crossing, after promotion to the $\mathbf{D}$ state, will occur over a much longer range of pump-probe delay times when compared with promotion to the $\mathbf{C}$ state. This is further evidenced by the excited state absorption signal reaching a maximum just to the blue of $5 \mathrm{eV}$ which lies directly in the energetic region of the $\mathbf{C}-\mathbf{D}$ avoided crossing.

\subsection{Nonadiabatic Dynamics}

Comparisons between quantum and SQC/MM population dynamics, when the wavepacket is promoted to the $\mathbf{C}$ state, are shown in the left and middle panels of Fig. 6. The contour lines represent a twenty percent decrease in population of the initially populated $\mathbf{C}$ state. Over the 250 fs pump-probe delay range, there are three regions that incur substantial population change $(\delta=0-20, \delta=70-120$, and $\delta=160-210)$. For pump-probe delay times $\delta<20$ fs the population transfer is rapid, between $t=10$ and $t=25 \mathrm{fs}$, ending with 

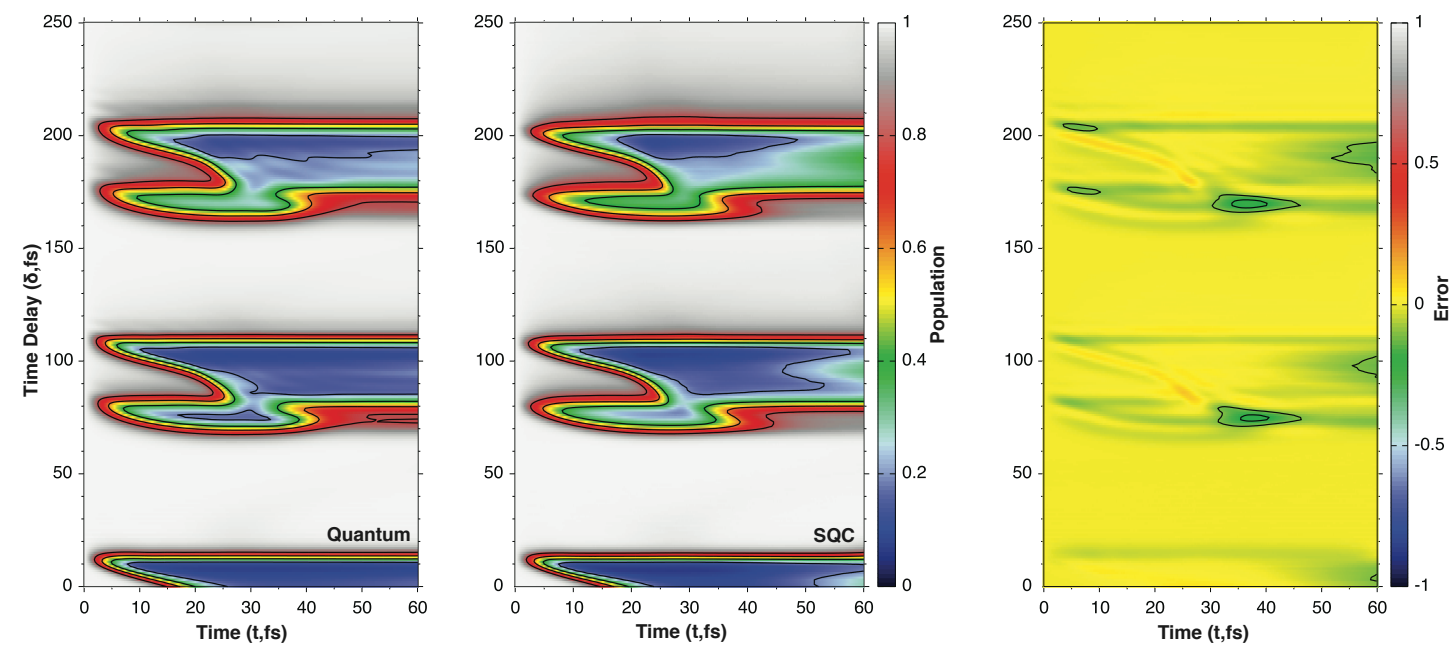

Fig. 6: The population dynamics from the $\mathbf{C}$ to the $\mathbf{D}$ adiabatic states simulated up to $t=60$ fs with pump-probe delay times $(\delta)$ between $0-250$ fs. The populations are calculated from quantum wavepacket dynamics (left) and the SQC/MM model (middle). The contour lines represent a decrease of twenty percent population change in each plot. The calculated signed error between the quantum and SQC/MM population dependence with contour lines representing a signed error of \pm 0.1 (right) .
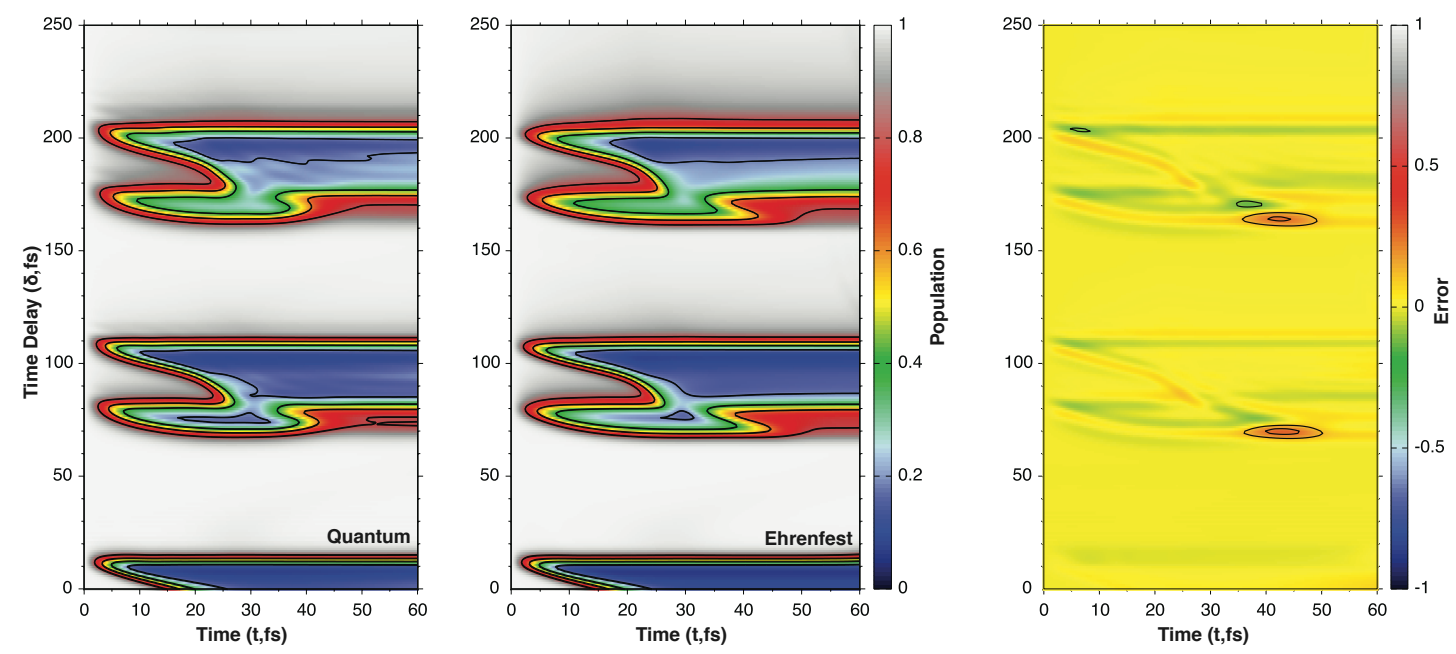

Fig. 7: The population dynamics from the $\mathbf{C}$ to the $\mathbf{D}$ adiabatic states simulated up to $t=60 \mathrm{fs}$ with pump-probe delay times $(\delta)$ between $0-250$ fs. The populations are calculated from quantum wavepacket dynamics (left) and the Ehrenfest model (middle). The contour lines represent a decrease of ten percent population change in each plot. The calculated signed error between the quantum and Ehrenfest population dependence with contour lines representing a signed error of \pm 0.1 (right) . 

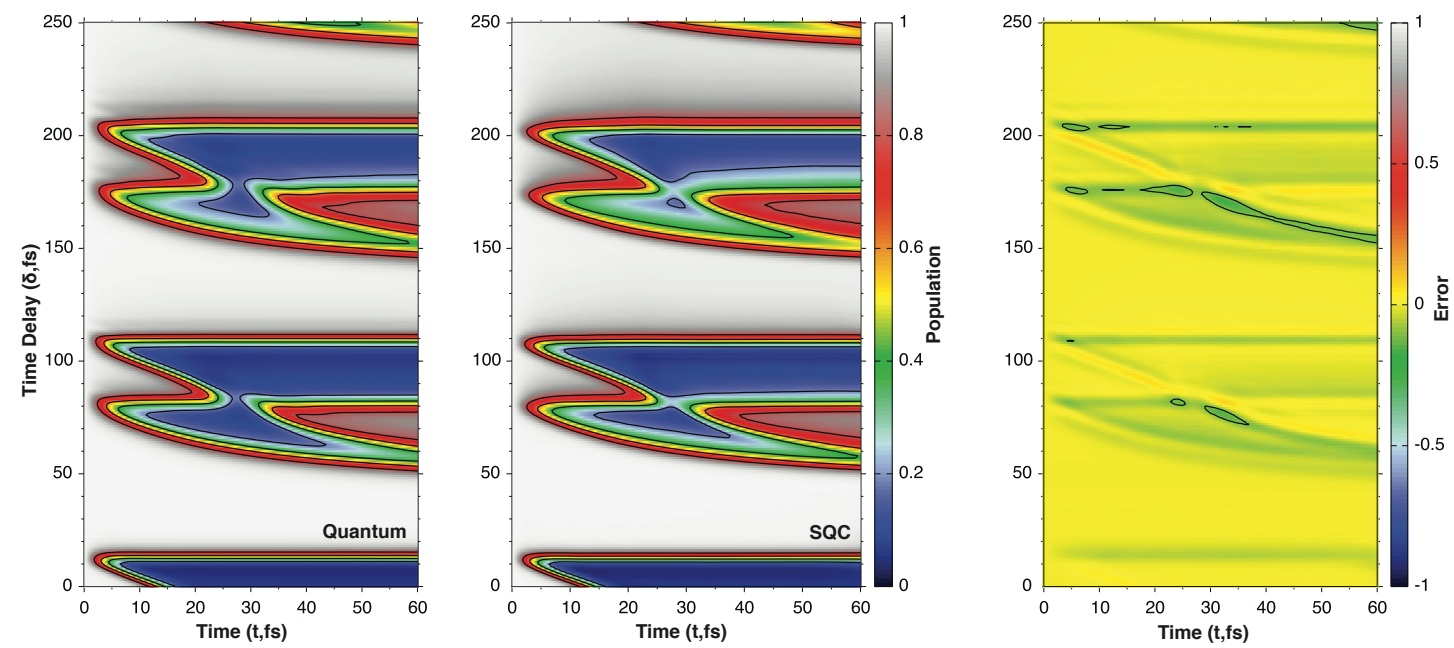

Fig. 8: The population dynamics from the $\mathbf{D}$ to the $\mathbf{C}$ adiabatic states simulated up to $t=60$ fs with pump-probe delay times $(\delta)$ between $0-250$ fs. The populations are calculated from quantum wavepacket dynamics (left) and the SQC/MM model (middle). The contour lines represent a decrease of ten percent population change in each plot. The calculated signed error between the quantum and SQC/MM population dependence with contour lines representing a signed error of \pm 10 percent (right) .
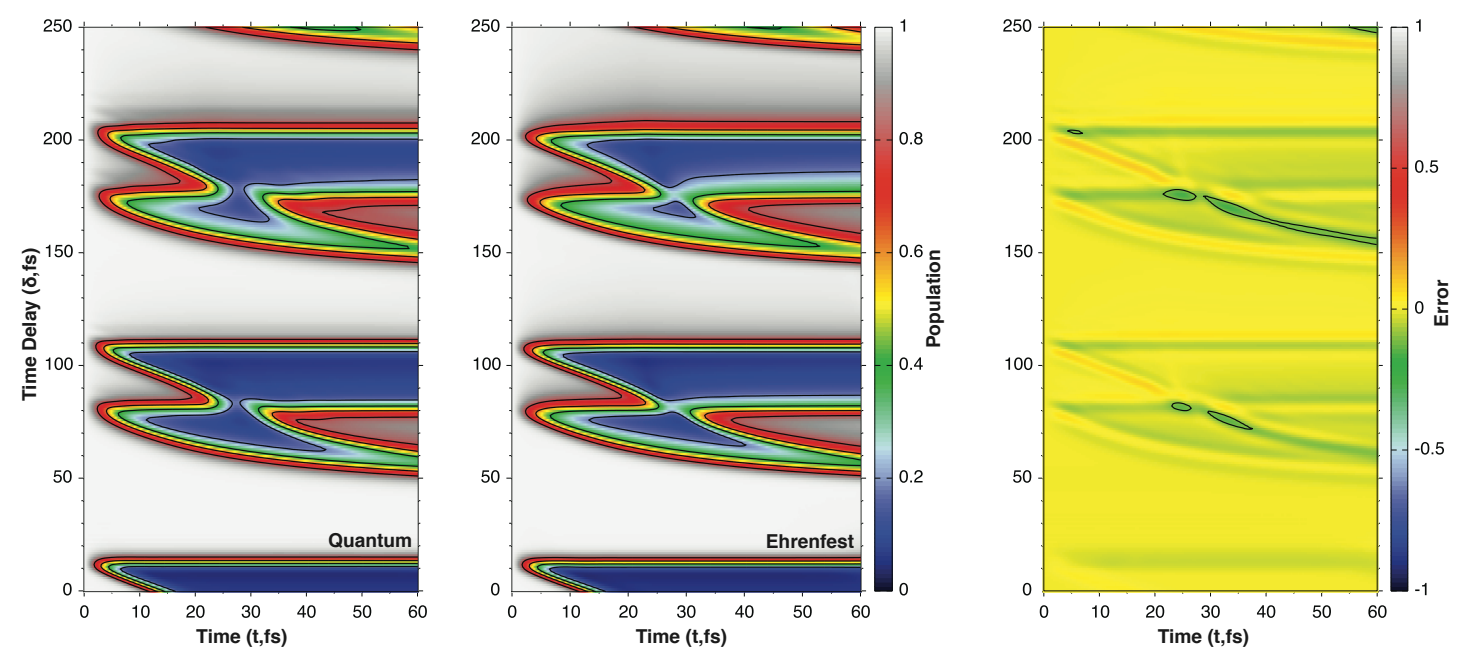

Fig. 9: The population dynamics from the $\mathbf{D}$ to the $\mathbf{C}$ adiabatic states simulated up to $t=60$ fs with pump-probe delay times $(\delta)$ between $0-250 \mathrm{fs}$. The populations are calculated from quantum wavepacket dynamics (left) and the Ehrenfest model (middle). The contour lines represent a decrease of ten percent population change in each plot. The calculated signed error between the quantum and Ehrenfest population dependence with contour lines representing a signed error of \pm 10 percent (right) . 

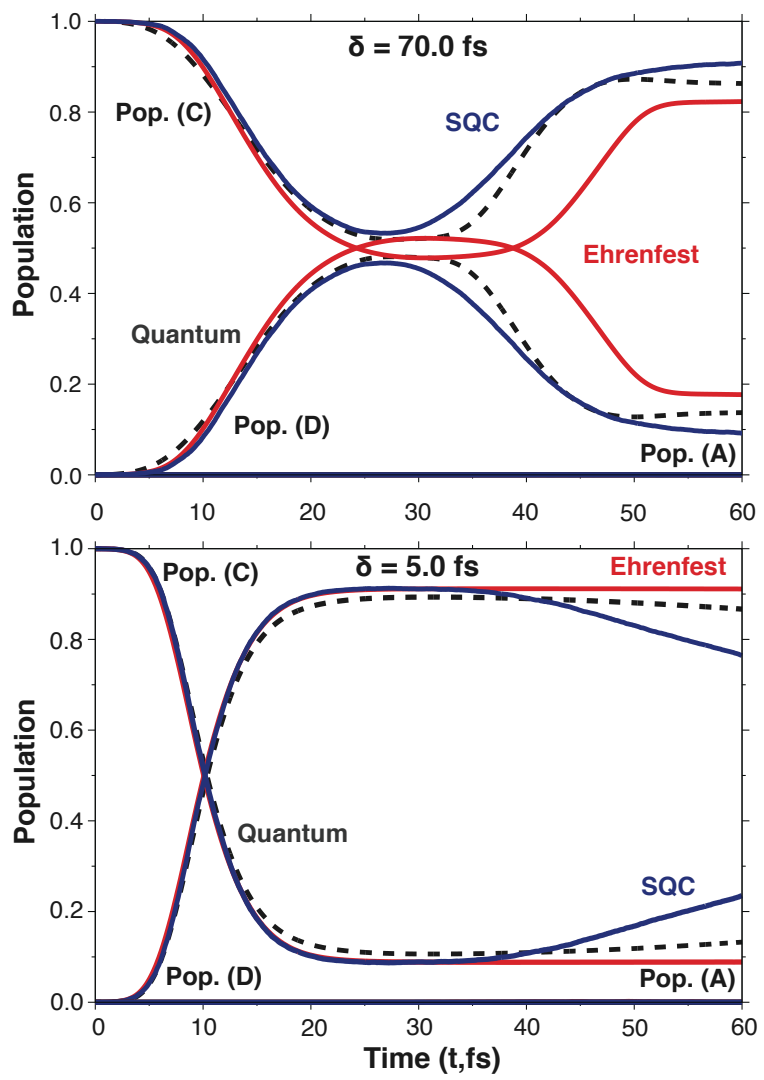

Fig. 10: Population dynamics at select pump-probe delay times $(\delta=5$ fs (bottom) and $\delta=70$ fs (top)) for population transfer from the $\mathbf{C}$ adiabatic state. Comparisons are made between Ehrenfest predictions (red), SQC/MM predictions (blue), and the quantum benchmark (black).

less than twenty percent population remaining in the $\mathbf{C}$ state. There are regions of pumpprobe delay times, however where there is no population change as the wavepacket leaves the nonadiabatically coupled region and approaches the outer well of the A potential energy surface. As the wavepacket approaches the avoided crossing from the right, which occurs first near $\delta=70 \mathrm{fs}$, the population transfer is again rapid with most of the population transferring before $t=10 \mathrm{fs}$. Finally, for $\delta>160 \mathrm{fs}$, the introduction of nodes in the wavepacket, propagating along the A surface, and the increased spread of the wavepacket results in an overall loss of efficiency in population transfer ending with less than thirty percent population remaining in the $\mathbf{C}$ state after passing through the avoided crossing region.

In general, the signed errors, which are simply defined as the difference between the 
quantum and SQC/MM results, are at most \pm 20 percent for population transfer from the $\mathbf{C}$ and $\mathbf{D}$ adiabatic states (see right panel of Fig. 6). The contour lines in the error plot represent a change in signed error of ten percent population change. The errors are more pronounced for longer simulation times ( $t>50 \mathrm{fs}$ ), as well as longer pump-probe delay times $(\delta>160 \mathrm{fs})$ with the largest errors occurring, in general, during the final pass through the avoided crossing. While the population transfer estimates with SQC/MM are slightly less efficient when compared with the quantum mechanical simulations, the SQC/MM dynamics do recover the same qualitative trend. For example, there is no population transfer when the initially excited classical particle is out of the nonadiabatically coupled region. Also consistent with the quantum mechanical simulations, the population transfer begins very quickly when the wavepacket approaches the avoided crossing from the left or the right (near $\delta=80,110,170$, and $200 \mathrm{fs}$ ) but is significantly slower when the wavepacket is promoted directly onto the avoided crossing region (near $\delta=0,90$ and $185 \mathrm{fs}$ ).

Comparisons between population transfer calculated with the Ehrenfest model and the quantum benchmark are provided in Fig. 7. While the signed errors between SQC/MM and Ehrenfest are comparable across most pump-probe delay times $\delta$, slightly lower errors are seen with Ehrenfest better estimating population dynamics in the longer time limit $(\delta>50 \mathrm{fs})$ across all three regimes. In fact, for population transfer from the $\mathbf{C}$ to $\mathbf{D}$ states errors with the Ehrenfest model are only greater than ten percent when the avoided crossing is approached from the right ( $\delta=70,160 \mathrm{fs}$ ). Interestingly, the errors in this region are positive where Ehrenfest tends to systematically overestimate the amount of population transfer compared with SQC/MM. For a clearer comparison of the error, two single cuts through the 2D population maps in Fig. 6 and 7 are shown, for $\delta=5$ fs and $\delta=70 \mathrm{fs}$, in Fig. 10.

When the wavepacket, or classical particle, is promoted to the $\mathbf{D}$ state, the population transfer is more efficient overall and occurs over a longer range of pump-probe delay times when compared with promotion to the $\mathbf{C}$ state. Comparisons between quantum wavepacket and SQC/MM population dynamics for this case are shown in left and middle panels of Fig. 
8. Again, the contour lines represent twenty percent decrease in population of the $\mathbf{D}$ state. Quantum mechanically the population transfer is very rapid with nearly eighty percent of the population occurring in $t<15$ fs - substantially shorter than population transfer from $\mathbf{C}$ to $\mathbf{D}$ which takes $t \approx 30$ fs. Also, when initially promoted to the $\mathbf{D}$ state, ranges of pump-probe delay times with no population transfer are shorter compared with population transfer from the $\mathbf{C}$ state which is consistent with the transient absorption spectrum being comprised of either primarily $\mathbf{D}$ vibronic states or states near the $\mathbf{C}-\mathbf{D}$ avoided crossing (see the top panel of Fig. 5). For longer pump-probe delay times $(\delta>150 \mathrm{fs})$, the population transfer quantum mechanically is again less efficient overall when compared with population transfer when $\delta>50$ fs and is more pronounced when the wavepacket approaches the avoided crossing from the right.

A similar agreement between the quantum mechanical and SQC/MM results is seen for population transfer from the $\mathbf{D}$ to $\mathbf{C}$ states with SQC/MM predicting population dynamics that are remarkably close to the quantum benchmark. Signed errors for this case are shown in the right panel of Fig. 8. In general, the errors are largest when the avoided crossing is approached from the right but still remain low over all pump-probe delay times. Comparable to the population dynamics from the $\mathbf{C}$ to the $\mathbf{D}$ states, the errors progressively worsen at longer pump-probe delay times with the largest errors occurring for $\delta>150 \mathrm{fs}$. When compared with the right panel of Fig. 6, where errors of twenty percent are seen when the avoided crossing is approached from the right, errors of only ten percent for this case are seen for pump-probe delay times $\delta>50$ and $\delta>150$ fs. Likewise for simulation times $t>50 \mathrm{fs}$, the errors are less than ten percent across all three relevant ranges of pump-probe delay times.

Comparisons between population transfer from the $\mathbf{D}$ to $\mathbf{C}$ states calculated with the Ehrenfest model and the quantum benchmark are provided in Fig. 9. The errors with Ehrenfest compared with SQC/MM are comparable when the population transfer occurs from the $\mathbf{D}$ to the $\mathbf{C}$ adiabatic states with the errors less than or equal to ten percent across all pump-probe delay times. 


\section{Conclusions}

In this work, quantitatively accurate potential energy surfaces, analytic nuclear gradients, and first-derivative couplings, calculated at the EOM-EE-CCSD level with a quadruple-zeta quality core-valence polarized basis set, were presented for the low-lying singlet states of sodium hydride. The vibronic energy levels of the ground and three excited electronic states were analyzed ( $\mathbf{X}, \mathbf{A}, \mathbf{C}$, and $\mathbf{D})$ using a variational eigensolver, directly in the adiabatic basis - which included the effect of first-order derivative coupling. As first-order derivative coupling between the $\mathbf{A}-\mathbf{C}$ and $\mathbf{A}-\mathbf{D}$ adiabatic potential energy surfaces had a negligible effect on the nonadiabatically coupled eigenstates, the nonadiabatic vibronic levels were effectively reduced to an electronic two-state problem involving only the $\mathbf{C}$ and $\mathbf{D}$ states. Between the $\mathbf{C}$ and $\mathbf{D}$ adiabatic states, the majority of population transfer occurred from a sharply localized but strong first-order derivative coupling that arises directly from the outer well of the $\mathbf{C}$ potential energy surface taking on substantial ionic character. In order to elucidate the effect this first-order derivative coupling had on the resulting eigenstates, the dependence on bond length was presented, as well as, the degradation of nonadiabatic character that maturates by expanding the eigenstates directly in the adiabatic basis.

Using the calculated nonadiabatically coupled vibronic energy levels, the transient absorption spectrum and quantum/quasi-classical nonadiabatic dynamics were analyzed by simulating a pump-probe type experiment. In this simulated experiment classical trajectories and a quantum wavepacket, moving along the A potential energy surface, was promoted to either the $\mathbf{C}$ or $\mathbf{D}$ adiabatic states at selected pump-probe delay times. For the transient absorption spectrum, the Franck-Condon overlap integrals were calculated and the difference spectrum was presented. Then, using the corresponding eigenstates, quantum/quasi-classical population dynamics were simulated and analyzed after time propagating the promoted wavepacket quantum mechanically and with classical trajectories. The exact quantum benchmarks were compared against predictions from the Ehrenfest and SQC/MM models which had signed errors of less than ten percent over most pump-probe 
delay times with errors around twenty percent only when the avoided crossing was approached from the right and for $t>30$ fs. In general, we found population transfer between the $\mathbf{C}$ and $\mathbf{D}$ states occurs ultrafast, between $t=15$ and $t=30 \mathrm{fs}$, depending on the initially excited wavepacket with more efficient population transfer occurring after promotion to the D state.

\section{Acknowledgements}

This work is supported by the Director, Office of Science, Office of Basic Energy Sciences of the US Department of Energy under contract No. DE-AC02-05CH11231. This work is supported by the National Science Foundation under grant number CHE-1856707.

\section{References}

(1) D. R. Yarkony, Rev. Mod. Phys., 1996, 68, 985.

(2) M. S. Schuurman and A. Stolow, Annu. Rev. Phys. Chem., 2018, 69, 427-450.

(3) B. G. Levine and T. J. Martínez, Annu. Rev. Phys. Chem., 2007, 58, 613-634.

(4) W. Domcke, D. R. Yarkony and H. Köppel, Conical intersections: theory, computation and experiment, World Scientific, 2011, vol. 17.

(5) A. W. Jasper, C. Zhu, S. Nangia and D. G. Truhlar, Faraday Discuss., 2004, 127, $1-22$.

(6) S. Matsika and P. Krause, Annu. Rev. Phys. Chem., 2011, 62, 621-643.

(7) J. E. Subotnik, J. Vura-Weis, A. J. Sodt and M. A. Ratner, J. Phys. Chem. A., 2010, 114, 8665-8675.

(8) Y. Shu and D. G. Truhlar, J. Chem. Theory Comput., 2020, 16, 6456-6464.

(9) Y. Shu, Z. Varga, A. G. Sampaio de Oliveira-Filho and D. G. Truhlar, J. Chem. Theory Comput., 2021, 17, 1106-1116. 
(10) Y. Mao, A. Montoya-Castillo and T. E. Markland, J. Chem. Phys., 2020, 153, 244111.

(11) S. L. Li, D. G. Truhlar, M. W. Schmidt and M. S. Gordon, J. Chem. Phys., 2015, 142, 064106.

(12) X. Zhang and J. M. Herbert, J. Chem. Phys., 2014, 141, 064104.

(13) J. E. Subotnik, E. C. Alguire, Q. Ou, B. R. Landry and S. Fatehi, Acc. Chem. Res., 2015, 48, 1340-1350.

(14) S. Fatehi, E. Alguire, Y. Shao and J. E. Subotnik, J. Chem. Phys., 2011, 135, 234105.

(15) S. Fatehi and J. E. Subotnik, J. Phys. Chem. Lett., 2012, 3, 2039-2043.

(16) J. M. Herbert, X. Zhang, A. F. Morrison and J. Liu, Acc. Chem. Res., 2016, 49, 931-941.

(17) J. C. Tully, J. Chem. Phys., 2012, 137, 22A301.

(18) B. F. E. Curchod and T. J. Martínez, Chem. Rev., 2018, 118, 3305-3336.

(19) U. N. Morzan, P. E. Videla, M. B. Soley, E. T. Nibbering and V. S. Batista, Angew. Chem., 2020, 132, 20219-20223.

(20) Y. Kobayashi, K. F. Chang, T. Zeng, D. M. Neumark and S. R. Leone, Science, 2019, 365, 79-83.

(21) H. Timmers, X. Zhu, Z. Li, Y. Kobayashi, M. Sabbar, M. Hollstein, M. Reduzzi, T. J. Martínez, D. M. Neumark and S. R. Leone, Nat. Commun., 2019, 10, 1-8.

(22) S. J. Cotton and W. H. Miller, J. Phys. Chem. A., 2013, 117, 7190-7194.

(23) S. J. Cotton and W. H. Miller, The Journal of chemical physics, 2013, 139, 234112.

(24) S. J. Cotton, R. Liang and W. H. Miller, J. Chem. Phys., 2017, 147, 064112.

(25) S. J. Cotton and W. H. Miller, J. Chem. Phys., 2019, 150, 194110.

(26) S. J. Cotton and W. H. Miller, J. Chem. Phys., 2019, 150, 104101.

(27) S. J. Cotton and W. H. Miller, J. Chem. Phys., 2016, 145, 144108.

(28) W. H. Miller and S. J. Cotton, Faraday Discuss., 2017, 195, 9-30. 
(29) H.-D. Meyera) and W. H. Miller, J. Chem. Phys., 1979, 70, 3214-3223.

(30) S. J. Cotton and W. H. Miller, J. Chem. Theory Comput., 2016, 12, 983-991.

(31) R. Liang, S. J. Cotton, R. Binder, R. Hegger, I. Burghardt and W. H. Miller, J. Chem. Phys., 2018, 149, 044101.

(32) D. Hu, Y. Xie, J. Peng and Z. Lan, J. Chem. Theory Comput., 2021, 3267-3279.

(33) D. Tang, W.-H. Fang, L. Shen and G. Cui, Phys. Chem. Chem. Phys., 2019, 21, $17109-17117$.

(34) J. S. Sandoval C, A. Mandal and P. Huo, J. Chem. Phys., 2018, 149, 044115.

(35) W. Zhou, A. Mandal and P. Huo, J. Phys. Chem. Lett., 2019, 10, 7062-7070.

(36) W. C. Stwalley, W. T. Zemke and S. C. Yang, J. Phys. Chem. Ref. Data, 1991, 20, $153-187$.

(37) S. R. Langhoff, C. W. Bauschlicher Jr and H. Partridge, J. Chem. Phys., 1986, 85, $5158-5166$.

(38) H.-Y. Huang, T.-L. Lu, T.-J. Whang, Y.-Y. Chang and C.-C. Tsai, J. Chem. Phys., 2010, 133, 044301.

(39) P. Fuentealba, O. Reyes, H. Stoll and H. Preuss, J. Chem. Phys., 1987, 87, 53385345.

(40) A. Belyaev, P. S. Barklem, A. Dickinson and F. Gadéa, Phys. Rev. A., 2010, 81, 032706 .

(41) R. W. Numrich and D. G. Truhlar, J. Phys. Chem., 1975, 79, 2745-2766.

(42) N. Khelifi, J. Phys. Chem. A., 2009, 113, 8425-8433.

(43) S. Faraji, S. Matsika and A. I. Krylov, J. Chem. Phys., 2018, 148, 044103.

(44) A. Tajti and P. G. Szalay, J. Chem. Phys., 2009, 131, 124104.

(45) T. Ichino, J. Gauss and J. F. Stanton, J. Chem. Phys., 2009, 130, 174105.

(46) M. Aymar, J. Deiglmayr and O. Dulieu, Can. J. Phys., 2009, 87, 543-556. 
(47) S.-D. Walji, K. M. Sentjens and R. J. Le Roy, J. Chem. Phys., 2015, 142, 044305.

(48) T. Rivlin, L. Lodi, S. N. Yurchenko, J. Tennyson and R. J. Le Roy, Mon. Not. R. Astron. Soc., 2015, 451, 634-638.

(49) C.-C. Chu, H.-Y. Huang, T.-J. Whang and C.-C. Tsai, J. Chem. Phys., 2018, 148, 114301.

(50) H.-Y. Huang, Y.-Y. Chang, M.-H. Liao, K.-L. Wu, T.-L. Lu, Y.-Y. Chang, C.-C. Tsai and T.-J. Whang, Chem. Phys. Lett., 2010, 493, 53-56.

(51) Y. Shao, Z. Gan, E. Epifanovsky, A. T. Gilbert, M. Wormit, J. Kussmann, A. W. Lange, A. Behn, J. Deng, X. Feng, D. Ghosh, M. Goldey, P. R. Horn, L. D. Jacobson, I. Kaliman, R. Z. Khaliullin, T. Kuś, A. Landau, J. Liu, E. I. Proynov, Y. M. Rhee, R. M. Richard, M. A. Rohrdanz, R. P. Steele, E. J. Sundstrom, H. L. W. III, P. M. Zimmerman, D. Zuev, B. Albrecht, E. Alguire, B. Austin, G. J. O. Beran, Y. A. Bernard, E. Berquist, K. Brandhorst, K. B. Bravaya, S. T. Brown, D. Casanova, C.-M. Chang, Y. Chen, S. H. Chien, K. D. Closser, D. L. Crittenden, M. Diedenhofen, R. A. D. Jr., H. Do, A. D. Dutoi, R. G. Edgar, S. Fatehi, L. Fusti-Molnar, A. Ghysels, A. Golubeva-Zadorozhnaya, J. Gomes, M. W. Hanson-Heine, P. H. Harbach, A. W. Hauser, E. G. Hohenstein, Z. C. Holden, T.-C. Jagau, H. Ji, B. Kaduk, K. Khistyaev, J. Kim, J. Kim, R. A. King, P. Klunzinger, D. Kosenkov, T. Kowalczyk, C. M. Krauter, K. U. Lao, A. D. Laurent, K. V. Lawler, S. V. Levchenko, C. Y. Lin, F. Liu, E. Livshits, R. C. Lochan, A. Luenser, P. Manohar, S. F. Manzer, S.-P. Mao, N. Mardirossian, A. V. Marenich, S. A. Maurer, N. J. Mayhall, E. Neuscamman, C. M. Oana, R. Olivares-Amaya, D. P. O’Neill, J. A. Parkhill, T. M. Perrine, R. Peverati, A. Prociuk, D. R. Rehn, E. Rosta, N. J. Russ, S. M. Sharada, S. Sharma, D. W. Small, A. Sodt, T. Stein, D. Stück, Y.-C. Su, A. J. Thom, T. Tsuchimochi, V. Vanovschi, L. Vogt, O. Vydrov, T. Wang, M. A. Watson, J. Wenzel, A. White, C. F. Williams, J. Yang, S. Yeganeh, S. R. Yost, Z.-Q. You, I. Y. Zhang, X. Zhang, Y. Zhao, B. R. Brooks, G. K. Chan, D. M. Chipman, C. J. Cramer, W. A. G. III, M. S. Gordon, 
W. J. Hehre, A. Klamt, H. F. S. III, M. W. Schmidt, C. D. Sherrill, D. G. Truhlar, A. Warshel, X. Xu, A. Aspuru-Guzik, R. Baer, A. T. Bell, N. A. Besley, J.-D. Chai, A. Dreuw, B. D. Dunietz, T. R. Furlani, S. R. Gwaltney, C.-P. Hsu, Y. Jung, J. Kong, D. S. Lambrecht, W. Liang, C. Ochsenfeld, V. A. Rassolov, L. V. Slipchenko, J. E. Subotnik, T. V. Voorhis, J. M. Herbert, A. I. Krylov, P. M. Gill and M. Head-Gordon, Mol. Phys., 2015, 113, 184-215.

(52) C. C. Marston and G. G. Balint-Kurti, J. Chem. Phys., 1989, 91, 3571-3576. 